\title{
What factors determine the need for lumbar puncture in patients with fever and headache?
}

\author{
Ki Joong $\underline{\mathrm{Kim}}^{1}$, MD, Jun Hwi $\underline{\mathrm{Cho}}^{1}$, MD, Joong Bum Moon ${ }^{1}$, MD, Myoung Cheol Shin ${ }^{1}$, MD, Taek Geun Ohk ${ }^{1}$, MD,
} Hui Young $\underline{L e e}^{2}, M D$, Chan Woo $\underline{\text { Park }}^{1}$, MD

INTRODUCTION We performed this study to find clinical features and laboratory parameters that could facilitate the process of selecting patients who should receive lumbar punctures from among those who present with headache and fever. METHODS We selected patients aged $\geq 16$ years who presented to and received lumbar puncture in the emergency department of Kangwon National University Hospital, South Korea, between 2011 and 2013. Patients who received lumbar punctures were divided into two groups - those who were diagnosed with viral meningitis and those who were not. We compared the clinical features and laboratory data between the two groups. Key indices were then used to develop a scoring system to diagnose viral meningitis in patients and identify those who should receive lumbar punctures.

RESULTS Among the patients who were included in the study, 42 had viral meningitis and 96 did not. The variables of C-reactive protein level $\leq 1.291 \mathrm{mg} / \mathrm{dL}$, neck stiffness and vomiting were assigned 3 points, 2 points and 1 point, respectively, in the scoring system. Overall scores $\geq 4$ yielded a positive likelihood ratio of 7.79 (sensitivity 0.600 , specificity 0.923), while negative likelihood ratio decreased to less than $0.1(0.072)$ for overall scores $<3$.

CONCLUSION Using the proposed scoring system, we were able to determine the likelihood of viral meningitis in patients presenting with fever and headache, and to successfully identify those who should receive lumbar punctures.

Keywords: fever, headache, lumbar puncture, meningitis

\section{INTRODUCTION}

Headaches may occur in a number of disorders. When both headache and fever are present in patients, meningitis is one of the conditions that should be considered. In the emergency department (ED), many patients receive lumbar punctures to diagnose or rule out meningitis. Although lumbar punctures are performed for patients who have fever of unknown cause or severe headache associated with fever, the results of cerebrospinal fluid (CSF) analyses are frequently not suggestive of meningitis. Lumbar punctures can be painful and require the patient to remain in the ED for several hours. There are also potential complications associated with the procedure, such as post-spinal tap headaches, which can lead to a certain degree of discomfort for patients. ${ }^{(1-6)}$ Therefore, screening is typically performed for all patients before lumbar punctures are performed, but is not required for other blood tests.

Symptoms such as the presence of neurological manifestations, neck stiffness and severe headache are used to screen for bacterial meningitis among patients who present with headache and fever. ${ }^{(7)}$ In the ED, it is not difficult to decide whether a lumbar puncture should be performed on patients diagnosed with bacterial meningitis, as the symptoms are relatively severe and frequently associated with neurological signs. The more common dilemma that clinicians face in the ED is whether to perform the procedure on patients presenting with viral meningitis-like symptoms. This is because it is challenging to clinically differentiate between patients with viral meningitis and those without meningitis, as the latter frequently present with symptoms that mimic viral meningitis..$^{(7-10)}$
We conducted the present study to find clinical features and laboratory parameters that could facilitate the selection process of patients who should receive lumbar punctures among those who present to the ED with headache and fever. We proposed to develop a new scoring system based on significant associations identified among key indices, which could be used to identify such patients.

\section{METHODS}

This was a retrospective case-control study approved by the institutional review board of Kangwon National University Hospital, Chuncheon-si, Korea. We selected all patients aged $\geq 16$ years who presented to and received lumbar punctures in the ED of Kangwon National University Hospital between 2011 and 2013. The hospital, situated in a mid-sized city with a population of approximately 300,000, has an annual ED attendance of 30,000-33,000. The indications for lumbar puncture were a change in mental status of unknown cause and suspected meningitis. In this study, lumbar punctures were performed either by emergency physicians or neurologists.

Patients who received lumbar punctures were further divided into two groups - those who were diagnosed with viral meningitis and those who were not (i.e. patients who had viral meningitislike symptoms but no meningitis). The initial diagnosis was made based on CSF analysis, including microscopic examinations (cell count, cell differential, Gram staining and acid-fast staining), chemical tests (protein and glucose), infectious disease tests (latex agglutination and polymerase chain reaction) and CSF culture, but the final diagnosis was made by a neurologist. 


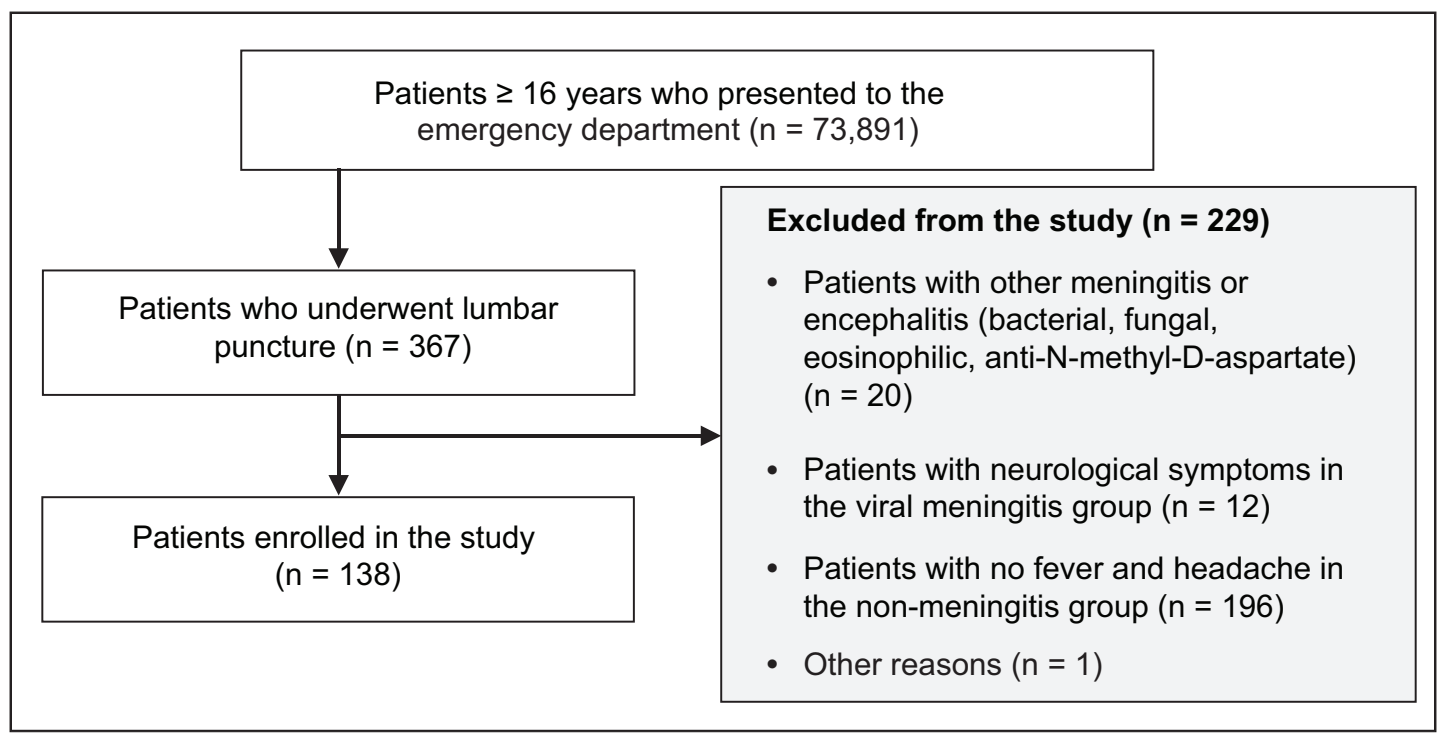

Fig. 1 Flowchart shows the patient selection process.

Among patients who had no viral meningitis, only those who initially presented with both fever and headache as their primary symptoms were included in the study. Among patients with viral meningitis, those with neurological symptoms were excluded from the study, as lumbar puncture was clearly indicated for these patients (Fig. 1). Patients who presented with bacterial meningitis were also considered to require lumbar puncture and excluded from the study. The final diagnosis of bacterial meningitis was made by a neurologist based on the clinical presentations and CSF results. Ten patients had positive CSF cultures or CSF Gram stains, one patient tested positive on CSF antigen testing and two patients had positive blood cultures. Brain computed tomography was conducted for all patients prior to the performance of lumbar punctures.

We compared the clinical features and laboratory data between the two patient groups (i.e. those with viral meningitis and those without). A retrospective analysis was performed on available data obtained from the hospital's electronic medical records. Clinical symptoms that were included in the analysis were fever, nausea, vomiting, diarrhoea, myalgia, photophobia, sleep disturbances, upper respiratory tract infection (URTI) symptoms and neck stiffness. Symptoms that were most frequently demonstrated by patients with and without viral meningitis were identified.

Blood tests that were performed included complete blood count with differential, aspartate aminotransferase, alanine aminotransferase, total bilirubin, gamma-glutamyl transpeptidase, lactate dehydrogenase (LDH), creatine phosphokinase (CPK) and $\mathrm{C}$-reactive protein (CRP). Blood tests that showed abnormal findings for infectious disease were selected. The ADVIA $® 1800$ Clinical Chemistry System (Siemens Healthineers, Deerfield, IL, USA), which uses latex-enhanced immunoturbidimetric assays, was used to measure CRP levels.

Comparison of clinical features was performed using chisquare test. Comparison of fever (i.e. body temperature in ${ }^{\circ} \mathrm{C}$ ) and laboratory data was performed using Student's $t$-test. The degree of attribution of each variable was assessed using multiple logistic regression analysis. The associations identified were used to develop a scoring system that could be used to diagnose viral meningitis in patients presenting to the ED with headache and fever, and to identify those who should receive lumbar punctures. IBM SPSS Statistics version 21.0 (IBM Corp, Armonk, NY, USA) software was used for statistical analysis.

\section{RESULTS}

Among patients aged $\geq 16$ years who presented to the ED during the study period, 54 patients with viral meningitis and 292 patients without meningitis received lumbar punctures. Among the 54 patients with viral meningitis, those with clear indications for lumbar puncture based on neurological symptoms $(n=12)$ were excluded. Hence, a total of 42 patients with viral meningitis were included in the study. Among patients without meningitis, only those who had both fever and headache as primary symptoms $(n=96)$ were included. The clinical features and laboratory findings for the two patient groups are listed in Tables I and II, respectively. For the majority of patients, symptoms such as diarrhoea, myalgia, photophobia and sleep disturbance were missing from the medical records; consequently, these symptoms were excluded from the analysis.

There was an equal number of men and women $(n=21$ each) among the 42 included patients with viral meningitis. The mean age of these patients was $34.7 \pm 14.1$ years and their mean body temperature was $37.4^{\circ} \mathrm{C} \pm 0.82^{\circ} \mathrm{C}$. Among the 96 included patients with no meningitis, 54 were men and 42 were women. Their mean age was $52.7 \pm 21.1$ years and mean body temperature was $38.0^{\circ} \mathrm{C} \pm 0.98^{\circ} \mathrm{C}$. The diagnoses for patients without meningitis are listed in Table III.

On univariate analysis, significant differences were found between the patient groups with and without viral meningitis for fever, nausea, vomiting, neck stiffness and URTI symptoms. Significant differences between the two groups were also found for these laboratory variables - neutrophil, lymphocyte and platelet counts, and LDH, CPK and CRP levels. 
Table I. Demographic and clinical features of patients with or without viral meningitis.

\begin{tabular}{lccc}
\hline \multirow{2}{*}{ Variable } & \multicolumn{2}{c}{ No. (\%) } & p-value \\
\cline { 2 - 3 } & With $(\mathbf{n}=\mathbf{4 2})$ & Without $(\mathbf{n}=\mathbf{9 6})$ & \\
\hline Age $(\mathrm{yr})^{*}$ & $34.7 \pm 14.1$ & $52.7 \pm 21.1$ & $<0.001$ \\
Male gender & $21(50.0)$ & $54(56.3)$ & 0.498 \\
Nausea $^{\dagger}$ & $29 / 37(78.4)$ & $35 / 66(53.0)$ & 0.011 \\
Vomiting $^{\dagger}$ & $18 / 37(48.6)$ & $15 / 61(24.6)$ & 0.015 \\
Neck stiffness $^{\dagger}$ & $22 / 38(57.9)$ & $12 / 48(25.0)$ & 0.002 \\
URTI symptoms $^{\dagger}$ & $2 / 33(6.1)$ & $18 / 53(34.0)$ & 0.003 \\
Fever $\left.^{\circ}{ }^{\circ} \mathrm{C}\right)^{*}$ & $37.4 \pm 0.82$ & $38.0 \pm 0.98$ & $<0.001$ \\
\hline
\end{tabular}

*Data presented as mean \pm standard deviation. +Data was missing for some patients and calculations were based on stated no. of available patients. URTI: upper respiratory tract infection

Table II. Laboratory findings of patients with or without viral meningitis.

\begin{tabular}{lccc}
\hline Variable & \multicolumn{2}{c}{ Mean \pm standard deviation } & p-value \\
\cline { 2 - 3 } & With $(\mathbf{n}=\mathbf{4 2})$ & Without $(\mathbf{n}=\mathbf{9 6})$ & \\
\hline White blood & $7.8 \pm 2.6$ & $8.8 \pm 5.1$ & 0.119 \\
cells (103/mL) & & & \\
$\quad$ Neutrophil (\%) & $66.5 \pm 12.4$ & $75.1 \pm 13.4$ & 0.001 \\
Lymphocyte (\%) & $24.1 \pm 9.8$ & $16.7 \pm 11.3$ & $<0.001$ \\
Platelet (103/mL) & $242 \pm 70$ & $180 \pm 85$ & $<0.001$ \\
AST (U/L) & $29 \pm 14$ & $73 \pm 285^{*}$ & 0.312 \\
ALT (U/L) & $26 \pm 21$ & $53 \pm 178^{*}$ & 0.331 \\
LDH (U/L) & $176 \pm 104^{+}$ & $339 \pm 257^{*}$ & $<0.001$ \\
CPK (U/L) & $75 \pm 60^{\S}$ & $170 \pm 180^{*}$ & 0.001 \\
CRP (mg/dL) & $0.588 \pm 0.971$ & $6.792 \pm 7.059$ & $<0.001$ \\
\hline
\end{tabular}

Data calculated based on: *95 patients, $\$ 17$ patients, $\$ 59$ patients and $\S 19$ patients. ALT: alanine aminotransferase; AST: aspartate aminotransferase; CPK: creatine phosphokinase; CRP: C-reactive protein; LDH: lactate dehydrogenase

Vomiting, neck stiffness and CRP level were chosen as diagnostic indices (Table IV). For the purpose of developing a scoring system for diagnosing viral meningitis, these three variables were represented as proper values of regression coefficients. The cut-off value for CRP level was determined to be $1.291 \mathrm{mg} / \mathrm{dL}$ (sensitivity 0.813; specificity 0.833; area under the curve [AUC] 0.897; Fig. 2). The regression coefficients were calculated by performing multiple logistic regression analysis, and the numeric values, being the score for each diagnostic factor, were designated by excluding the decimal points. CRP level $\leq 1.291 \mathrm{mg} / \mathrm{dL}$ was scored as 3 points, neck stiffness as 2 points and vomiting as 1 point (Table IV). Overall scores $\geq 3$ yielded a positive likelihood ratio $(\mathrm{LR}+$ ) of 4.60 (sensitivity: 0.943; specificity: 0.795) (Table V) and overall scores $\geq 4$ yielded an LR+ of 7.79 (sensitivity: 0.600; specificity: 0.923). On the other hand, a negative likelihood ratio (LR-) decreased to less than 0.1 (0.072) when the score was $<3$. When we applied a diagnostic index to each of the patients, the AUC of the receiver operating characteristic curve was 0.908 (95\% confidence interval 0.839-0.977; Fig. 3).

A total of 17 patients $(12$ male, five female; age $60.0 \pm$ 21.9 years) with bacterial meningitis presented to the $\mathrm{ED}$ during the study period. Among these, 12 patients had neurological
Table III. Diagnoses of patients without viral meningitis $(n=96)$.

\begin{tabular}{|c|c|}
\hline Diagnosis & No. \\
\hline Fever & 19 \\
\hline Viral infection & 8 \\
\hline Headache & 5 \\
\hline Infectious colitis & 5 \\
\hline Tonsillitis & 5 \\
\hline Acute gastroenteritis & 4 \\
\hline Pneumonia & 4 \\
\hline Sinusitis & 4 \\
\hline Urinary tract infection & 4 \\
\hline Acute pyelonephritis & 3 \\
\hline Pharyngitis & 3 \\
\hline Upper respiratory tract infection & 3 \\
\hline Vascular headache & 3 \\
\hline Other specified headache syndrome & 3 \\
\hline Hantavirus haemorrhagic fever with renal syndrome & 2 \\
\hline Hepatitis & 2 \\
\hline Influenza & 2 \\
\hline Tension headache & 2 \\
\hline Tsutsugamushi disease & 2 \\
\hline Acute infectious mononucleosis & 1 \\
\hline Bacteraemia & 1 \\
\hline Cellulitis & 1 \\
\hline Cholangiohepatitis & 1 \\
\hline Cholecystitis & 1 \\
\hline Kikuchi disease & 1 \\
\hline Leptospirosis & 1 \\
\hline Middle cerebral artery aneurysm & 1 \\
\hline Migraine & 1 \\
\hline Septic knee & 1 \\
\hline Soft tissue infection & 1 \\
\hline Subacute bacterial endocarditis & 1 \\
\hline Tick bite & 1 \\
\hline
\end{tabular}

symptoms and five patients had neck stiffness without neurological symptoms. The mean CRP level among the patients with bacterial meningitis was $7.949 \pm 8.136 \mathrm{mg} / \mathrm{dL}$ (Table VI).

\section{DISCUSSION}

Meningitis should be considered when both fever and headache are present in a patient. In our study, a number of patients presented to our ED with fever and headache as the primary complaints.

For patients with bacterial meningitis, a diagnostic lumbar puncture is mandatory, as life-threatening complications may occur. ${ }^{(11,12)}$ While most patients with viral meningitis improve with symptomatic therapy, complications may occur, such as those seen among patients with herpes simplex virus encephalitis, and therefore a lumbar puncture is also required to diagnose viral meningitis. ${ }^{(13-19)}$ Hence, a lumbar puncture is often performed to screen for meningitis, especially among patients who present with fever and headache. Even after meningitis is ruled out following a lumbar puncture, the patient must be observed in the 
Table IV. Assigning of scores based on regression coefficients using multiple logistic regression analysis.

\begin{tabular}{lcccc}
\hline Variable & Regression coefficient $(\boldsymbol{\beta})$ & Standard error & p-value & OR (95\% Cl) \\
\hline CRP $\leq 1.291 \mathrm{mg} / \mathrm{dL}$ & 3.679 & 0.841 & $<0.001$ & $39.623(7.627-205.852)$ \\
Neck stiffness & 2.074 & 0.838 & 0.013 & $7.960(1.541-41.125)$ \\
Vomiting & 1.549 & 0.747 & 0.038 & $4.705(1.089-20.335)$ \\
\hline
\end{tabular}

$\mathrm{Cl}$ : confidence interval; CRP: C-reactive protein; OR: odds ratio

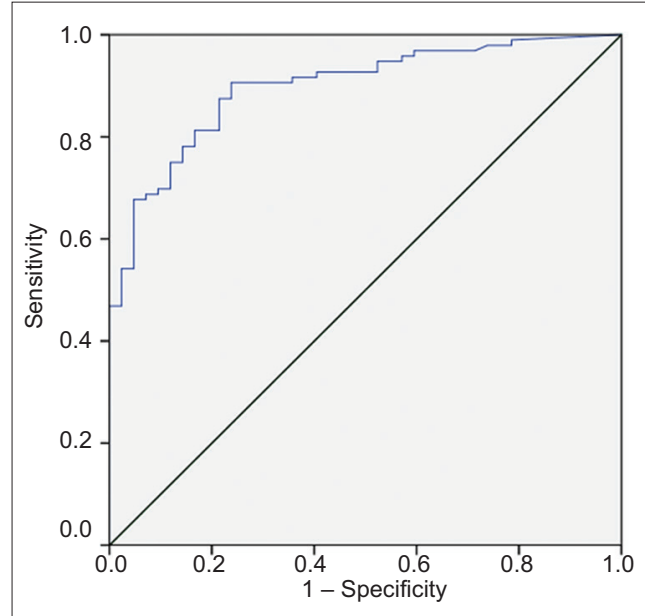

Fig. 2 Receiver operating characteristic curve of C-reactive protein (area under curve 0.897; 95\% confidence interval 0.845-0.950).

Table V. Performance of index score for predicting viral meningitis.

\begin{tabular}{lcccc}
\hline Score & Sensitivity & Specificity & LR+ & LR- \\
\hline$\geq 0$ & 1.000 & 0.000 & 1.00 & NA \\
$\geq 1$ & 0.971 & 0.513 & 1.99 & 0.057 \\
$\geq 2$ & 0.971 & 0.590 & 2.37 & 0.049 \\
$\geq 3$ & 0.943 & 0.795 & 4.60 & 0.072 \\
$\geq 4$ & 0.600 & 0.923 & 7.79 & 0.433 \\
$\geq 5$ & 0.400 & 0.974 & 15.38 & 0.616 \\
$\geq 6$ & 0.171 & 1.000 & NA & 0.829 \\
\hline
\end{tabular}

LR+: positive likelihood ratio; LR-: negative likelihood ratio; NA: not available

ED for several hours, as complications from the procedure may occasionally arise, causing discomfort to the patient. ${ }^{(1-6)}$

An absence of neurological symptoms is not uncommon in patients with viral meningitis, creating challenges in clinically differentiating between these patients and those with viral meningitis-like symptoms (but not meningitis). ${ }^{(7-10)}$ Although Brudzinski's or Kernig's signs have been used to clinically distinguish between the two groups, the sensitivity of these approaches is low. ${ }^{(20-22)}$ As it is difficult to make a clear clinical distinction, the decision-making process of whether to perform a lumbar puncture on patients presenting with both fever and headache can be challenging. Notwithstanding this, few studies exist in the literature on the clinical differences between patients with viral meningitis and those with meningitis-like symptoms.

Given the paucity of relevant literature and established guidelines, this study aimed to develop a scoring system to identify patients who require lumbar puncture among those presenting to the ED with headache and fever. For patients with bacterial meningitis, the majority of whom have neurological symptoms

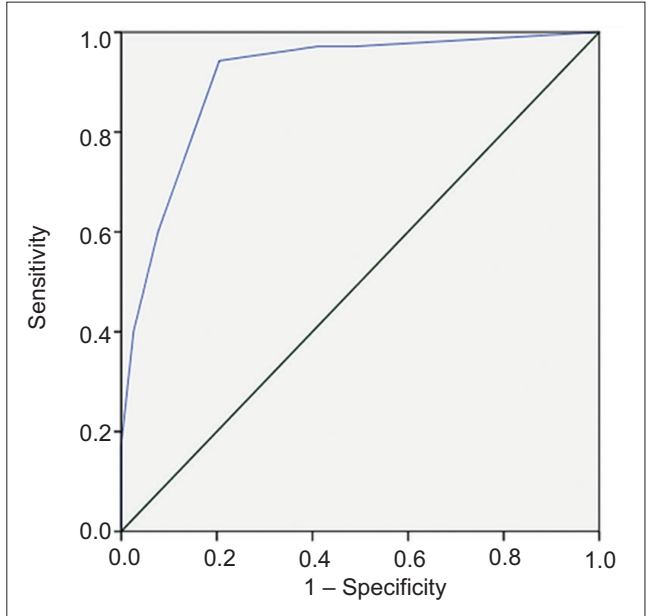

Fig. 3 Receiver operating characteristic curve of diagnostic index (area under curve 0.908; 95\% confidence interval 0.839-0.977).

Table VI. C-reactive protein (CRP) level of patients with bacterial meningitis.

\begin{tabular}{llc}
\hline No. & Gender/age $(\mathbf{y r})$ & CRP $(\mathbf{m g} / \mathbf{d L})^{*}$ \\
\hline 1 & Female/74 & 32.794 \\
2 & Female/75 & 21.211 \\
3 & Male/75 & 11.785 \\
4 & Male/84 & 10.560 \\
5 & Female/76 & 7.726 \\
6 & Male/70 & 7.410 \\
7 & Male/86 & 7.116 \\
8 & Male/86 & 6.383 \\
9 & Female/27 & 6.338 \\
10 & Female/59 & 5.981 \\
11 & Male/67 & 5.638 \\
12 & Male/41 & 5.301 \\
13 & Male/45 & 3.454 \\
14 & Male/39 & 1.263 \\
15 & Male/69 & 1.100 \\
16 & Male/32 & 1.060 \\
17 & Male/19 & 0.020 \\
\hline
\end{tabular}

*Mean CRP: $7.949 \pm 8.136 \mathrm{mg} / \mathrm{dL}$.

and severe headache, it is not difficult for clinicians to decide whether a lumbar puncture is indicated. In our study, 17 patients who presented to the ED with bacterial meningitis were excluded from the analysis, as no significant barriers were encountered in determining the need for lumbar punctures.

We observed differences in the presence of symptoms, such as nausea, vomiting, neck stiffness, URTI symptoms and fever, between patients with viral meningitis and those without. There were also differences in laboratory variables, such as neutrophil, 
lymphocyte and platelet counts, and LDH, CPK and CRP levels. In order to devise a scoring system that might help to determine the need for a lumbar puncture, this study included vomiting, neck stiffness and CRP level as diagnostic indices, because these variables were found to be markedly different between patients with and without viral meningitis in the clinical setting. In our study, the AUC was 0.897 (sensitivity 0.813, specificity 0.833) for a CRP cut-off value of $1.291 \mathrm{mg} / \mathrm{dL}$ (Fig. 2). We also observed that patients with viral meningitis characteristically had lower CRP levels than those with meningitis-like symptoms, such that CRP level by itself demonstrated diagnostic utility $(p<0.001)$. The mean CRP level of patients who had no meningitis was $6.792 \pm 7.059 \mathrm{mg} / \mathrm{dL}$.

The scoring system in our study was established by assigning 3 points to CRP levels $\leq 1.291 \mathrm{mg} / \mathrm{dL}$, 2 points to neck stiffness and 1 point to vomiting. With overall score $\geq 4, \mathrm{LR}+$ was over 5 (LR+ 7.79), and with overall score $\geq 5, L R+$ was over 10 ( $L R+15.38)$. LR- was less than $0.1(0.072)$ when the score was $<3$. This suggests that there is an increased possibility of a diagnosis of viral meningitis at overall scores $\geq 4$ and a considerably higher possibility at overall scores $\geq 5$, with a greater likelihood of ruling out viral meningitis at overall scores $<3$.

An advantage of our proposed scoring system is that it can be easily applied in everyday clinical practice. Photophobia and neurological symptoms, which are infrequent among patients with viral meningitis, were not included in the scoring system, augmenting its usefulness in determining the need for lumbar puncture among patients with meningitis-like symptoms in the practical setting. However, this scoring system could not be applied to patients with bacterial, tuberculous or fungal meningitis. The proposed scoring system should not be used to diagnose meningitis but to obtain additional information when deciding whether lumbar puncture should be performed on patients with meningitis-like symptoms, especially in cases of possible viral meningitis.

The present study was a retrospective, case-control study, as meningitis is a relatively uncommon condition. Validation of the proposed scoring system is warranted by way of future prospective studies. Nevertheless, by using the scoring system for patients presenting to the ED with fever and headache, we were able to determine the possibility of viral meningitis (i.e. aseptic meningitis) among these patients and successfully identify those who should receive lumbar punctures. By assigning 3 points to CRP levels $\leq 1.291 \mathrm{mg} / \mathrm{dL}, 2$ points to neck stiffness and 1 point to vomiting, there was a high possibility of diagnosing viral meningitis and ascertaining the need for lumbar puncture in patients with overall scores $\geq 4$. This scoring system could potentially help to reduce the number of lumbar punctures performed, potential complications associated with the procedure and patient length of stay in the ED, and ultimately bring down the attendant costs of healthcare.

\section{REFERENCES}

1. Headache Classification Subcommittee of the International Headache Society. The International Classification of Headache Disorders: 2nd edition. Cephalalgia 2004; 24 Suppl 1:9-160.

2. Peskind ER, Riekse R, Quinn JF, et al. Safety and acceptability of the research lumbar puncture. Alzheimer Dis Assoc Disord 2005; 19:220-5.

3. Alcolea D, Martínez-Lage $P$, Izagirre A, et al. Feasibility of lumbar puncture in the study of cerebrospinal fluid biomarkers for Alzheimer's disease: a multicenter study in Spain. J Alzheimers Dis 2014; 39:719-26.

4. Zetterberg $\mathrm{H}$, Tullhög $\mathrm{K}$, Hansson $\mathrm{O}$, et al. Low incidence of post-lumbar puncture headache in 1,089 consecutive memory clinic patients. Eur Neurol 2010; 63:326-30.

5. Blennow K, Wallin A, Häger O. Low frequency of post-lumbar puncture headache in demented patients. Acta Neurol Scand 1993; 88:221-3.

6. Vimala J, Peter JV, Jeyaseelan L, Prabhakar S, Cherian AM. Post lumbar puncture headache: is bed rest essential? J Assoc Physicians India 1998; 46:930-2.

7. van de Beek D, de Gans J, Spanjaard L, et al. Clinical features and prognostic factors in adults with bacterial meningitis. N Engl J Med 2004; 351:1849-59.

8. Rorabaugh ML, Berlin LE, Rosenberg L, et al. Absence of neurodevelopmental sequelae from aseptic meningitis. Pediatr Res 1992; 30:177A.

9. Magnussen CR. Meningitis in adults: ten-year retrospective analysis at a community hospital. N Y State J Med 1980; 80:901-6.

10. Behrman RE, Meyers BR, Mendelson MR, Sacks HS, Hirschman SZ. Central nervous system infections in the elderly. Arch Intern Med 1989; 149:1596-9.

11. Matthew PF. The nervous system. In: Abbas AK, Kumar V, Fausto N, Mitchell R, eds. Robbin's basic pathology. Philadelphia: Saunders Elsevier, 2007: 873-81.

12. Morton NS, Nath A. Meningitis: bacterial, viral, and other. In: Goldman L, Schafer Al, eds. Goldman's Cecil medicine. Philadelphia: Saunders Elsevier, 2012: 2355-70

13. De Tiège X, Rozenberg F, Héron B. The spectrum of herpes simplex encephalitis in children. Eur J Paediatr Neurol 2008; 12:72-81.

14. Granerod J, Ambrose HE, Davies NW, et al; UK Health Protection Agency (HPA) Aetiology of Encephalitis Study Group. Causes of encephalitis and differences in their clinical presentations in England: a multicentre, population-based prospective study. Lancet Infect Dis 2010; 10:835-44.

15. Marks DA, Kim J, Spencer DD, Spencer SS. Characteristics of intractable seizures following meningitis and encephalitis. Neurology 1992; 42:1513-8.

16. McGrath N, Anderson NE, Croxson MC, Powell KF. Herpes simplex encephalitis treated with acyclovir: diagnosis and long term outcome. J Neurol Neurosurg Psychiatry 1997; 63:321-6.

17. Stahl JP, Mailles A, De Broucker T; Steering Committee and Investigators Group. Herpes simplex encephalitis and management of acyclovir in encephalitis patients in France. Epidemiol Infect 2012; 140:372-81.

18. Trinka E, Dubeau F, Andermann F, et al. Clinical findings, imaging characteristics and outcome in catastrophic post-encephalitic epilepsy. Epileptic Disord 2000; 2:153-62.

19. Yoshioka M, Kuroki S, Mizue H. Clinical and electroencephalographic studies of postencephalitic epilepsy. Acta Paediatr Jpn 1989; 31:480-3.

20. Brody IA, Wilkins RH. The signs of Kernig and Brudzinski. Arch Neurol 1969; 21:215-8.

21. Uchihara T, Tsukagoshi $\mathrm{H}$. Jolt accentuation of headache: the most sensitive sign of CSF pleocytosis. Headache 1991; 31:167-71.

22. Thomas KE, Hasbun R, Jekel J, Quagliarello VJ. The diagnostic accuracy of Kernig's sign, Brudzinski's sign, and nuchal rigidity in adults with suspected meningitis. Clin Infect Dis 2002; 35:46-52. 